\title{
Akinetic Crisis in Parkinson's Disease Is Associated with a Severe Loss of Striatal Dopamine Transporter Function: A Report of Two Cases
}

\author{
Valtteri Kaasinen ${ }^{a, b} \quad$ Juho Joutsa ${ }^{b}$ Tommi Noponen ${ }^{c}$ \\ Markku Päivärinta ${ }^{d}$ \\ ${ }^{a}$ Division of Clinical Neurosciences, ${ }^{b}$ Turku PET Centre, and ${ }^{\mathrm{c} D e p a r t m e n t}$ of Clinical \\ Physiology and Nuclear Medicine, University of Turku and Turku University Hospital, \\ Turku, Finland; ${ }^{d}$ Visby Lasarett, Visby, Sweden
}

\section{Key Words}

Parkinson's disease $\cdot$ Dopamine transporter $\cdot$ SPECT $\cdot$ Akinesia

\begin{abstract}
Akinetic crisis or acute akinesia is a life-threatening complication of Parkinson's disease (PD) with unknown pathophysiological mechanisms. Clinically, it resembles the neuroleptic malignant syndrome, and dopaminergic drugs are transiently ineffective in the acute phase of the condition. There are no published dopaminergic functional imaging studies on PD patients with akinetic crisis. Here we report 2 advanced PD patients with akinetic crisis who were scanned with SPECT using brain dopamine transporter ligand $\left[{ }^{123} \mathrm{I}\right] \mathrm{FP}-\mathrm{CIT}$. The first patient was additionally scanned before the condition developed, and the second patient was scanned after recovery. Striatal dopamine transporter binding was lower during than before the crisis, and both patients showed a nearly complete loss of dopamine transporter binding during the crisis. Serial imaging showed that the uptake remained negligible despite an improvement in motor function after recovery. Akinetic crisis in PD appears to be associated with a particularly severe loss of presynaptic striatal dopamine function that does not improve after recovery. Apart from presynaptic dopaminergic function, other dopaminergic or nondopaminergic mechanisms are involved in the clinical improvement of motor functions after akinetic crisis in PD.




\section{Introduction}

Akinetic crisis or acute akinesia is a life-threatening complication of Parkinson's disease (PD) with an estimated annual incidence of $0.3 \%$ and a death rate of $15 \%[1,2]$. Akinetic crisis refers to a situation when motor symptoms of PD have acutely worsened, up to a point when the patient is nearly completely akinetic, often associated with dysphagia, hyperthermia, dysautonomia and/or increased levels of serum muscle enzymes [1]. The clinical picture is similar to that of neuroleptic malignant syndrome, and the condition has also been termed as neuroleptic malignant-like syndrome, malignant syndrome or parkinsonismhyperpyrexia [3]. Akinetic crisis can be triggered by a number of factors such as treatment manipulations or withdrawal from treatment, infections, traumas or gastrointestinal tract diseases $[1,4]$.

The pathophysiological mechanism of akinetic crisis is unclear. The condition does not seem to be merely a reflection of extreme parkinsonian hypokinesia, but rather a specific condition with unknown risk factors that are not directly related to disease stage or medication dosage [1]. One of the main features of akinetic crisis appears to be the longlasting (on average 11 days) unresponsiveness to anti-parkinsonian rescue medications such as subcutaneous apomorphine or nasogastric levodopa [1]. Therefore, the dopamine system during akinetic crisis appears to be transiently blocked from the treatments that usually give patients rapid motor benefit. However, there are no dopaminergic functional imaging studies or even reported cases with imaging in parkinsonian akinetic crisis. It is not known if patients that develop akinetic crisis have advanced dopaminergic degeneration that predisposes them to develop akinetic crisis, or if there are dynamic changes in the dopamine system that correlate with the dramatic clinical change in the recovery phase as the patient slowly regains mobility after extreme akinesia.

In the present paper, we describe 2 PD patients that developed akinetic crisis and were scanned twice with SPECT using dopamine transporter ligand [123I]FP-CIT.

\section{Case 1}

A 57-year-old man with a 12-year history of PD was admitted to our hospital due to constipation and intestinal pseudo obstruction as seen in abdominal X-ray examination. Diagnostic investigations also included laboratory tests [blood count, C-reactive protein (CRP), creatine phosphokinase (CPK), myoglobin, troponin, liver function, kidney function, glucose, electrolytes, cortisol, urine screen], electrocardiogram (ECG), chest X-ray and brain CT. He had Hoehn and Yahr stage 4 and had shown a good response to anti-parkinsonian drugs (treated with levodopa monotherapy $250 \mathrm{mg} /$ day at the time of admission). Two years before the onset of akinetic crisis, a [123I]FP-CIT SPECT had been performed for diagnostic confirmatory purposes. The scan showed a clear bilateral dopaminergic defect with nearly absent putaminal binding with a striatal-to-occipital ratio of 1.76 and 1.79 on the right and left hemisphere, respectively (fig. 1a).

Before the development of the akinetic crisis, the patient had decreased bowel movements for several weeks. The treating physician noted that he was very rigid and akinetic, and at times, he did not respond to questions. On admission, he was bed-ridden, drowsy, showed severe rigidity and akinesia, and had increased CPK of $463 \mathrm{I} / \mathrm{U}$ (normal $<280 \mathrm{I} / \mathrm{U}$ ) without fever and a slightly elevated plasma CRP value of $17 \mathrm{mg} / \mathrm{l}$ (normal $<10 \mathrm{mg} / \mathrm{l}$ ). Brain CT was normal. 
Akinetic crisis was suspected and levodopa treatment via nasogastric tube was initiated. After 2 weeks of nearly complete akinesia, a [123I]FP-CIT SPECT scan was performed, showing a very low uptake of the tracer with a striatal-to-occipital cortex ratio of 1.56 (right) and 1.33 (left) (fig. 1b). The dopaminergic defect had clearly progressed as compared to the scan 2 years earlier (fig. 1f). The tracer binding was calculated from investigator-drawn volumes of interest for all images using PMOD (version 3.2, PMOD Technologies, Zurich, Switzerland). Conventional BRASS analysis (Hermes Medical Solutions AB, Stockholm, Sweden) that is used in the clinical practice could not be performed reliably due to the very low tracer uptake in the striatum.

Intestinal pseudo-obstruction was successfully treated and the patient slowly regained mobility with a partial recovery 2 weeks after admission (wheel chair) and a full recovery after 6 months (walking with support).

\section{Case 2}

An 82-year-old man with a 5-year history of PD was admitted to our hospital due to severe akinesia. He was on Hoehn and Yahr stage 3 and had shown a good response to levodopa (treated with levodopa monotherapy $400 \mathrm{mg} /$ day combined with entacapone at the time of admission). Diagnostic investigations included laboratory tests (blood count, CRP, CPK, myoglobin, troponin, liver function, kidney function, thyroid function, glucose, electrolytes, calcium, urine screen), ECG, chest X-ray, brain CT and electroencephalography.

On admission, the patient had not taken his medication for several days. He was stuporous, incontinent, anarthric, and showed severe rigidity and akinesia. He was unable to walk and had a low blood pressure of 108/68 mm Hg. Plasma CPK on admission was 1,061 I/U, plasma myoglobin was also increased at $443 \mu \mathrm{g} / \mathrm{l}$ (normal $<72 \mu \mathrm{g} / \mathrm{l}$ ) and the patient developed hyperthermia $\left(38.4^{\circ} \mathrm{C}\right)$ with a normal CRP of $2 \mathrm{mg} / \mathrm{l}$. A levodopa-treament was initiated via a nasogastric tube. Two weeks after admission, a partial recovery was noted (the patient was able to move his upper extremities), but he was still bed-ridden. At that point, a [123I]FP-CIT SPECT scan was performed showing a nearly complete loss of striatal tracer binding with a striatal-to-occipital ratio of 1.53 (right) and 1.58 (left) (fig. 1d). Three weeks after admission, the patient could sit on a wheel chair, and 1 month after admission, he was able to walk short distances with aid. Two months after admission, the patient was clinically nearly fully recovered; he was able to walk $30 \mathrm{~m}$ using support and he had normal speech. At that point, the second [123I]FP-CIT SPECT scan was performed (fig. 1e). The scan essentially showed the same finding (striatal-to-occipital cortex ratio of 1.56 on the right and 1.66 on the left hemisphere) as during the akinetic crisis and despite his clinical improvement (fig. 1f).

\section{Discussion}

We described 2 PD patients with akinetic crisis and serial dopamine transporter imaging. Dopamine transporter binding at the time of the akinetic crisis was negligible in both patients. There was no asymmetry in binding, which probably reflects a bilaterally severe impairment of the nigrostriatal dopamine function. This type of a nearly complete loss of striatal dopamine transporter binding has been termed as the 'burst striatum' [5] and, from an imaging point of view, is the end stage of PD. Based on these 2 patients, it appears that the catatonic-like state during akinetic crisis in PD is a condition that is associated with a 
particularly severe presynaptic dopamine dysfunction. Although UPDRS scores were unavailable, the motor improvement was dramatic as both patients regained mobility after weeks of extreme rigidity and akinesia.

Patient one was also scanned 2 years before the akinetic crisis, and the binding at that time was very low, but clearly higher (still showing identifiable striatal regions) compared to the later scan (fig. 1a). This suggests that the negligible dopamine function does not represent a constant trait, but a progressed state that could also be due to general disease progression. On the other hand, the case of patient two shows that the dopamine transporter binding remained very low even after the patient was able to walk 2 months after the akinetic crisis had started. This constant and severe pathological finding suggests that other dopaminergic or nondopaminergic mechanisms (extrinsic or intrinsic), apart from striatal presynaptic dopamine system function, are involved in the recovery from the akinetic crisis of PD.

Parkinsonian akinetic crisis may be a condition that is associated with severe dopaminergic degeneration, which is not only related to disease stage, but to other factors such as possible genetic predisposition. The reported incidence of akinetic crisis in genetic PD (POLG1, PINK1, LRRK2, GBA) appears high (21.2 vs. $0.3 \%$ in idiopathic PD) and a genetically driven mitochondrial dysfunction was suggested to be an underlying cause of akinetic crisis [6]. Interestingly, DAT binding in mitochondrial DNA abnormalities, such as in POLG mutations, seems to be very low even in the absence of parkinsonism [7].

If an akinetic PD patient with neuroleptic malignant syndrome-like features is not responding to apomorphine or to increments of the current dopaminergic drugs, the possibility of acute akinesia should be considered [8]. The patients usually do not respond for days or weeks to the nasogastric administration of anti-parkinsonian drugs or to subcutaneous apomorphine, and they mostly have normal levodopa kinetics [1,9]. Both of our patients showed a rapid deterioration of their motor symptoms, together with refractoriness to nasogastric levodopa for 2 weeks, and they both had elevated CPK and dysautonomic features with disturbances of consciousness. Only the second patient had hyperthermia, but akinetic crisis can occur without fever [1]. Although misdiagnoses in akinetic crisis are common [9], the clinical picture and the delay in treatment response of our patients indicate that both had the condition known as akinetic crisis or acute akinesia. It has been suggested that akinetic crisis is not predictable based on PD duration, stage or treatments [9]. Indeed, the age, the disease duration and the medication doses were very different between our patients, although both developed an akinetic crisis; the first was associated with intestinal obstruction, and the second with treatment withdrawal. Both patients had negligible dopamine transporter bindings at the time of the crisis. It cannot be excluded that the akinetic crises of our patients were partially due to insufficient levodopa enteral absorption. Subcutaneous apomorphine injections were not available in Finland as a treatment choice for PD when these 2 patients developed symptoms of akinetic crises. Therefore, levodopa via a nasogastric tube was used as the first line of treatment.

To conclude, based on these first 2 patients with dopaminergic imaging, an akinetic crisis is associated with bilaterally nearly absent striatal dopamine transporter bindings. The burst striatum seems to be a result of progression, but it is not a prognostic factor, and the uptake remains low despite clear improvement in motor function after recovery. 
Kaasinen et al.: Akinetic Crisis in Parkinson's Disease Is Associated with a Severe Loss of Striatal Dopamine Transporter Function: A Report of Two Cases

\section{Acknowledgements}

The staff of the Department of Nuclear Medicine, Turku University Hospital, is gratefully acknowledged.

\section{Disclosure Statement}

This study was financially supported by the Academy of Finland (decision No. 256836) and the Turku University Hospital (ERVA funds). On behalf of all authors, the corresponding author states that there is no conflict of interest.

\section{References}

1 Onofrj M, Thomas A: Acute akinesia in Parkinson disease. Neurology 2005;64:1162-1169.

-2 Takubo H, Harada T, Hashimoto T, Inaba Y, Kanazawa I, Kuno S, Mizuno Y, Mizuta E, Murata M, Nagatsu T, Nakamura S, Yanagisawa N, Narabayashi H: A collaborative study on the malignant syndrome in Parkinson's disease and related disorders. Parkinsonism Relat Disord 2003;9(suppl 1):S31-S41.

-3 Toru M, Matsuda O, Makiguchi K, Sugano K: Neuroleptic malignant syndrome-like state following a withdrawal of antiparkinsonian drugs. J Nerv Ment Dis 1981;169:324-327.

4 Thomas A, Iacono D, Luciano AL, Armellino K, Onofrj M: Acute akinesia or akinetic crisis in Parkinson's disease. Neurol Sci 2003;24:219-220.

5 Eggers C, Kahraman D, Fink GR, Schmidt M, Timmermann L: Akinetic-rigid and tremor-dominant Parkinson's disease patients show different patterns of FP-CIT single photon emission computed tomography. Mov Disord 2011;26:416-423.

-6 Bonanni L, Onofrj M, Valente EM, Manzoli L, De Angelis MV, Capasso M, Thomas A: Recurrent and fatal akinetic crisis in genetic-mitochondrial parkinsonisms. Eur J Neurol 2014;21:1242-1246.

-7 Tzoulis C, Tran GT, Schwarzlmüller T, Specht K, Haugarvoll K, Balafkan N, Lilleng PK, Miletic H, Biermann M, Bindoff LA: Severe nigrostriatal degeneration without clinical parkinsonism in patients with polymerase gamma mutations. Brain 2013;136:2393-2404.

8 Thomas A, Onofrj M: Akinetic crisis, acute akinesia, neuroleptic malignant-like syndrome, Parkinsonismhyperpyrexia syndrome, and malignant syndrome are the same entity and are often independent of treatment withdrawal. Mov Disord 2005;20:1671.

-9 Onofrj M, Bonanni L, Cossu G, Manca D, Stocchi F, Thomas A: Emergencies in parkinsonism: akinetic crisis, life-threatening dyskinesias, and polyneuropathy during L-Dopa gel treatment. Parkinsonism Relat Disord 2009;15(suppl 3):S233-S236. 


\section{Case Reports in Neurology}

\begin{tabular}{l|l}
\hline Case Rep Neurol 2014;6:275-280 \\
\hline DOI: 10.1159/000369448 & $\begin{array}{l}\text { ○ 2014 S. Karger AG, Basel } \\
\text { www.karger.com/crn }\end{array}$ \\
\hline
\end{tabular}

Kaasinen et al.: Akinetic Crisis in Parkinson's Disease Is Associated with a Severe Loss of Striatal Dopamine Transporter Function: A Report of Two Cases

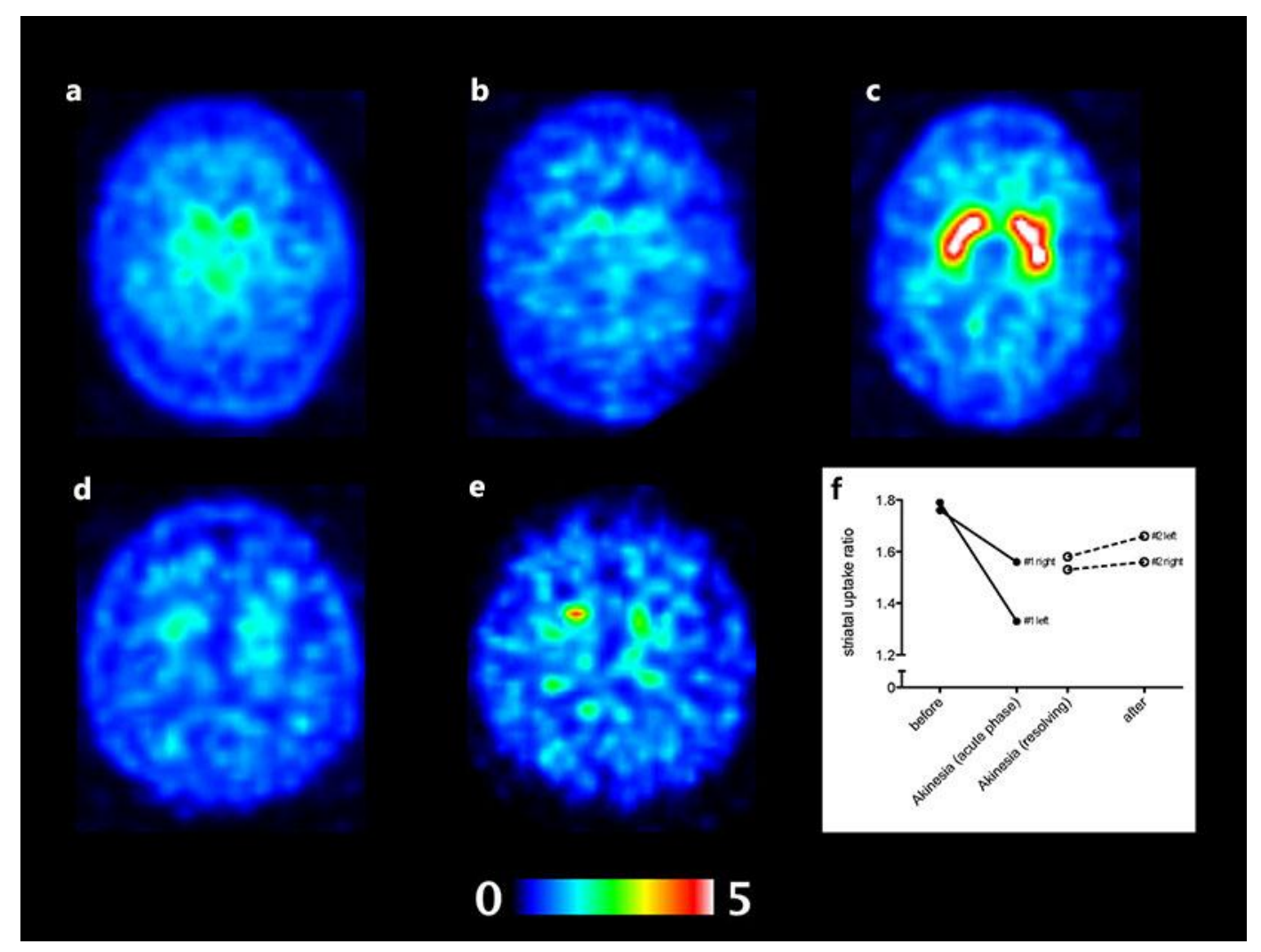

Fig. 1. [123I]FP-CIT SPECT region-to-occipital cortex ratio images. a Case 1 scanned before the akinetic crisis. b Case 1 scanned during the akinetic crisis. c Normal scan of a patient without nigrostriatal degeneration for comparison. d Case 2 scanned during the akinetic crisis. e Case 2 scanned after the akinetic crisis. $\mathbf{f}$ Longitudinal changes in striatal-to-occipital cortex binding ratio in case 1 (solid circles and lines) and case 2 (open circles and dotted lines). The color scale bar represents region-to-occipital cortex binding values. 